\title{
Instrumental and consummatory behavior as a function of length of reward-period
}

\author{
Roger W. Black and Patricia Elstad \\ STATE UNIVERSITY OF IOWA
}

\begin{abstract}
Three experiments are described in which rats were trained to run a straight alley, the reinforcement being 10 or $30 \mathrm{sec}$. access to wet mash. In each study, Ss trained with $10 \mathrm{sec}$. reward ran faster than $30 \mathrm{sec}$. Ss. In the second and third studies, in which food consumption during the reward-period was recorded, the $10 \mathrm{sec}$. Ss were found to eat, as well as run, faster than the 30 sec. group. Possible interpretations of these data are indicated.
\end{abstract}

\section{Problem}

Experiment 1 was designed for purposes unrelated to the present report. The results of that experiment, however, suggested that under some circumstances a nominally "small reward" may act as a more effective incentive than a "larger" reward. The subsequent investigations were conducted to determine the reproducibility of these initial results and to provide a basis for interpreting them.

\section{Method}

In Experiment 1, 18 male, hooded rats, about 130 days old, were trained to run a $3 \mathrm{ft}$ straight alley for food reward. The Ss were maintained on a diet of $8 \mathrm{gm}$ of standard laboratory chow and ad lib. water for 10 days before and throughout training. Four trials were given daily for 12 days with an intertrial interval of about $8 \mathrm{~min}$. For half the Ss, reinforcement on each trial was $30 \mathrm{sec}$. access to a caster in the goal box filled with wet mash, while for the other group the reward-period was $10 \mathrm{sec}$. Running speed was recorded electronically on each trial over the second 6 in of the alley. Experiment 2 constituted a replication of Experiment 1 with the exceptions that the Ss were 16 female, hooded rats, approximately 120 days old at the beginning of training and the amount of food eaten by each $S$ was measured on the last three days of training. Experiment 3 was a replication of Experiment 2 except that the amount eaten during the reward-period was recorded for each $\mathrm{S}$ throughout the experiment and the groups consisted of five female Ss each.

\section{Results}

Figure 1, representing acquisition running speeds for the combined experiments (i.e., 22 Ss per group), indicates that, at least over the final five days of training, the "small reward" (10 sec.) group run faster than the "large reward" (30 sec.) group. This superiority of the $10 \mathrm{sec}$. Ss was observed in each experiment and was statistically reliable over Days 7-11 for the combined data, $F(1,43)=5.11 ; p<.05$.

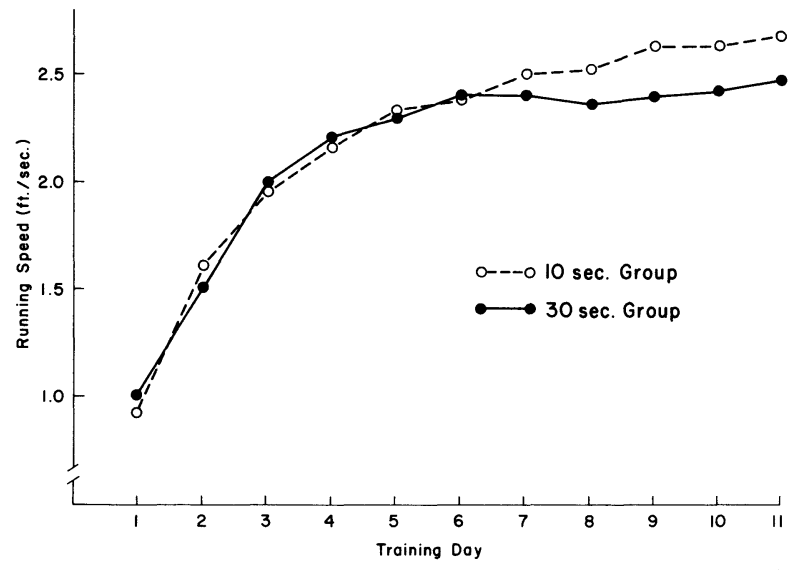

Fig. 1. Running speed as a function of daily blocks of four trials.

Figure 2 indicates "average rate of eating" in Experiment 3 (i.e., gms of food consumed per trial divided by the length of the reward-period). It is apparent that while average eating rates were initially equivalent for the two groups, the $10 \mathrm{sec}$. Ss came to subsequently eat at a substantially faster rate than the 30 sec. Ss. A similar effect was also observed on the last three days of the second experiment and in both cases the difference in eating rate on those days was statistically reliable, $t(14)=3.16 ; p<.01$ in the second, and $t(8)=3.72$; $\mathrm{p}<.01$ in the third experiment. Nevertheless, although

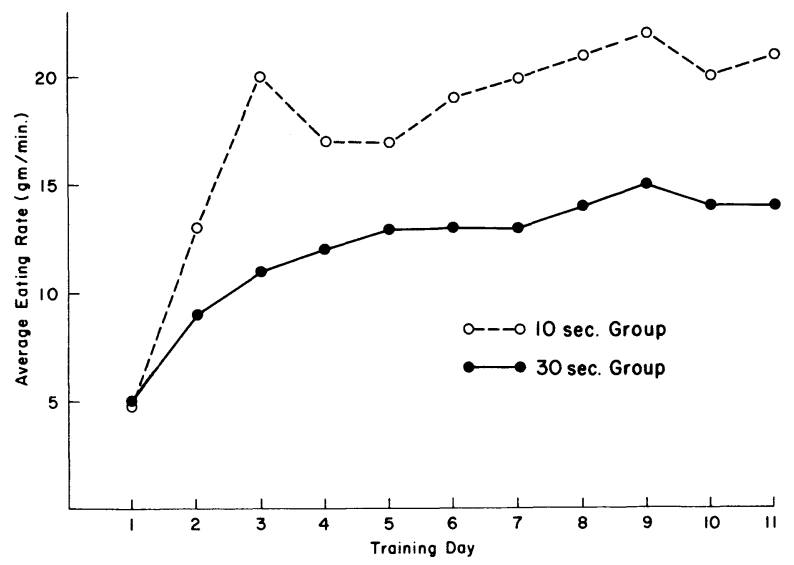

Fig. 2. Average eating rate as a function of daily blocks of four trials. 
Ss in the $10 \mathrm{sec}$. group showed a higher average rate of eating, Ss in the $30 \mathrm{sec}$. group consumed more food per trial (.31 gm vs. $.20 \mathrm{gm}$ per trial on Days 9-11).

\section{Discussion}

The primary results of the present experiments were that Ss trained with a 10 sec. reward-period appeared to perform both the consummatory $\mathbf{R}\left(\mathrm{R}_{\mathrm{G}}\right)$ and the instrumental $\mathbf{R}\left(\mathbf{R}_{\mathbf{i}}\right)$ more vigorously than Ss trained with a longer (30 sec.) reward-period. With respect to $R_{G}$, however, it is uncertain whether it was the actual $r$ at $e$ of eating-while eating-or merely the consistency of eating which was greater in the $10 \mathrm{sec}$.group. Observations of Ss' behavior in the goal box, however, strongly suggested that consistency of eating was at least one of the factors involved, since Ss in the $10 \mathrm{sec}$ group appeared to spend a much smaller percentage of their reward-period in nonconsummatory behavior than the $30 \mathrm{sec}$. Ss.

A possible interpretation of the fact that Ss in the 10 sec. group ate more vigorously or consistently is that with long reward-periods, nonconsummatory $\mathrm{Rs}$ in the goal box will have a relatively high probability of being reinforced (i.e., followed by an opportunity for further eating). With a shorter reward-period, there is increased probability that a nonconsummatory $\mathbf{R}$ will "terminate" the trial and, hence, not be reinforced. Thus, the acquisition of differential degrees of consistency of eating may indicate the extent to which nonconsummatory Rs have extinguished. If it is further assumed that such nonconsummatory Rs may generalize from the goal box to the runway and compete with $R_{i}$, then this interpretation would also account for the faster running of the $10 \mathrm{sec}$. Ss. On the other hand, if it is assumed that the "vigor" of $R_{G}$ was greater for the $10 \mathrm{sec}$. Ss than in the $30 \mathrm{sec}$. group, then the present results would also follow from Spence's (1956) interpretation of "incentive motivation" based on the strength of conditioned, implicit consummatory behavior $\left(\mathrm{r}_{\mathrm{g}}-\mathrm{s}_{\mathrm{g}}\right)$ and a related analysis of primary and secondary reinforcement suggested by Black (1964).

The finding that rats run faster to nominally "small rewards" than to "larger" ones is, of course, contrary to the result consistently reported in the psychological literature. It should be noted, however, that the present study employed both an atypical method of varying reward magnitude (eating-time rather than food-quantity) and a rather atypical maintenance procedure (fixedquantity of food rather than fixed-hours of deprivation). It is possible that Ss maintained on a fixed-eating-time schedule learn to eat rapidly or consistently as a consequence of that schedule, with the result that no rate-ofeating difference develops between short and long reward-period groups. A fixed-food-quantity schedule, however, might not be expected to produce such rapid or consistent eating behavior and, hence, different reward-periods would have the opportunity to result in different eating rates. The present finding may, thus, depend upon the interaction of the specific maintenance procedures employed and the method of varying " reward magnitude." In any event, research currently being conducted by the writers suggests the preliminary conclusion that the strength of both $R_{i}$ and $R_{G}$ are decreasing functions of the length of reward-period over a range of from 5 to $60 \mathrm{sec}$.

\section{References}

BLACK, R. W. Differential conditioning, extinction and secondary reinforcement. J. exp. Psychol., 1964, 68, in press.

SPENCE, K. W. Behavior theory and conditioning. New Haven: Yale Univer. Press, 1956. 